\title{
On delay-interval-dependent robust stability of LPD discrete-time system with mixed time-varying delays and nonlinear uncertainties
}

\author{
Kanit Mukdasai ${ }^{1}$ and Narongrit Kaewbanjak ${ }^{2^{*}}$ (1)
}

"Correspondence: srcnrk@ku.ac.th

2Faculty of Science at Sriracha, Kasetsart University Sriracha

Campus, Chon Buri, Thailand Full list of author information is available at the end of the article

\section{Springer}

\begin{abstract}
In this work, a delay-interval-dependent robust stability problem for linear parameter dependent (LPD) discrete-time system with discrete and distributed time-varying delays and nonlinear uncertainties is addressed. By adopting combinations of mixed model transformation, various inequality expressions, utilization of zero equations, and new parameter dependent Lyapunov-Krasovskii functional, a new delay-interval-dependent asymptotic stability criterion is derived in the form of linear matrix inequalities. Moreover, an improved delay-interval-dependent asymptotic stability criterion of discrete-time linear system with discrete time-varying delay and nonlinear uncertainties is also achieved. Some numerical examples are also illustrated to exhibit the effectiveness with less conservatism of the proposed stability criterion.
\end{abstract}

Keywords: Parameter dependence; Lyapunov-Krasovskii functional; Linear matrix inequality (LMI); LPD discrete-time system; Interval time-varying delay

\section{Introduction}

During the past decades, various stability problems of discrete-time systems with delays have drawn many researchers' attention, for delays in systems may lead to instability or bad performances [1-30]. Discrete-time systems with time-varying delays have been well recognized to be typical examples of applications to engineering [3]. In recent years, various stability analyses of both discrete- and continuous-time systems with respect to uncertainty about time-invariant parameters have been among many challenging problems. An important aspect of uncertainty in a linear system is a linear parameter-dependent (LPD) system in which the uncertain state matrices in the polytope are mathematically expressed by a convex combination of known matrices. Most stability criteria have been obtained via Lyapunov-Krasovskii functional approaches, in which parameter-dependent Lyapunov functions have been employed [1, 8, 13, 14, 25, 28]. Stability analysis for discrete-time systems with interval time-varying delay and nonlinear uncertainties was studied in [21]. The robust stability criteria of LPD continuous-time system with delays were presented in [28]. Some results have been obtained for delay-interval-dependent robust stability of discrete-time systems with discrete and distributed time-varying delays [27]. However,

(c) The Author(s) 2019. This article is distributed under the terms of the Creative Commons Attribution 4.0 International License (http://creativecommons.org/licenses/by/4.0/), which permits unrestricted use, distribution, and reproduction in any medium, provided you give appropriate credit to the original author(s) and the source, provide a link to the Creative Commons license, and indicate if changes were made. 
few results have been obtained for robust stability for LPD discrete-time systems with interval time-varying delays and nonlinear perturbations.

Motivated by the above statement, the problem of stability for LPD discrete-time systems with mixed interval time-varying delays and nonlinear uncertainties is studied. Through the construction of a parameter-dependent Lyapunov-Krasovskii functional, model transformation, various forms of inequalities and utilization of zero equations, a delay-interval-dependent robust stability criterion is presented in terms of linear matrix inequalities (LMIs) whose feasibility can be easily checked. Then, based on the derived delay-interval-dependent robust stability criterion, an improved delay-range-dependent stability criterion is proposed for a discrete-time system with interval discrete timevarying delay and nonlinear uncertainties. Moreover, we include some numerical examples to exhibit the effectiveness with less conservatism of the proposed stability criterion.

\section{Problem formulation}

Notations, definition and lemmas used throughout this paper are given as follows. By $Z^{+}$we denote the set of nonnegative integers; $R^{n}$ denotes the $n$-dimensional space with the vector norm $\|\cdot\|$; by $\|\xi\|$ we denote the Euclidean vector norm of $\xi \in R^{n}$, that is, $\|\xi\|^{2}=\xi^{T} \xi ; R^{n \times r}$ denotes the space of all $(n \times r)$-dimensional real matrices; $A^{T}$ denotes the transpose of a matrix $A$, while $I$ denotes the identity matrix. Matrix $A$ is called semipositive definite $(A \geq 0)$ if $x^{T} A x \geq 0$, for all $x \in R^{n} ; A$ is positive definite $(A>0)$ if $x^{T} A x>0$ for all $x \neq 0$. Matrix $B$ is called semi-negative definite $(B \leq 0)$ if $x^{T} B x \leq 0$, for all $x \in R^{n} ; B$ is negative definite $(B<0)$ if $x^{T} B x<0$ for all $x \neq 0$; $A>B$ means $A-B>0 ; A \geq B$ means $A-B \geq 0 ; \lambda(A)$ denotes the set of all eigenvalues of $A ; \lambda_{\max }(A)=\max \{\operatorname{Re} \lambda: \lambda \in \lambda(A)\}$; $\lambda_{\min }(A)=\min \{\operatorname{Re} \lambda: \lambda \in \lambda(A)\} ; \lambda_{\max }(A(\varphi))=\max \left\{\lambda_{\max }\left(A_{i}\right): i=1,2, \ldots, N\right\} ; \lambda_{\min }(A(\varphi))=$ $\min \left\{\lambda_{\min }\left(A_{i}\right): i=1,2, \ldots, N\right\}$, and $*$ represents the elements below the main diagonal of a symmetric matrix.

Consider the LPD discrete-time system with mixed interval time-varying delays and nonlinear perturbations of the form

$$
\begin{aligned}
& \xi(k+1)= A(\varphi) \xi(k)+B(\varphi) \xi(k-h(k))+C(\varphi) \sum_{i=1}^{\infty} \delta(i) \xi(k-i) \\
&+f_{1}(k, \xi(k))+f_{2}(k, \xi(k-h(k))) \\
& \xi(s)=\phi(s), \quad s \in\left\{-h_{2},-h_{2}+1, \ldots, 0\right\}
\end{aligned}
$$

where $\xi(k) \in R^{n}$ is the state variable for $k \in Z^{+}$and $\phi(s)$ is the initial value at $s ; A(\varphi), B(\varphi)$, $C(\varphi) \in R^{n \times n}$ stand for uncertain matrices defined by

$$
\begin{aligned}
& A(\varphi)=\sum_{i=1}^{N} \varphi_{i} A_{i}, \quad B(\varphi)=\sum_{i=1}^{N} \varphi_{i} B_{i}, \quad C(\varphi)=\sum_{i=1}^{N} \varphi_{i} C_{i}, \\
& \sum_{i=1}^{N} \varphi_{i}=1, \quad \varphi_{i} \geq 0, A_{i}, B_{i}, C_{i} \in R^{n \times n}, i=1, \ldots, N .
\end{aligned}
$$

The nonlinear perturbations $f_{1}(k, \xi(k))$ and $f_{2}(k, \xi(k-h(k)))$ are bounded in magnitude:

$$
f_{1}^{T}(k, \xi(k)) f_{1}(k, \xi(k)) \leq \gamma^{2} \xi^{T}(k) \xi(k),
$$




$$
f_{2}^{T}(k, \xi(k-h(k))) f_{2}(k, \xi(k-h(k))) \leq \beta^{2} x^{T}(k-h(k)) \xi(k-h(k))
$$

where $\gamma$ and $\beta$ are given positive real constants. The discrete time-varying delay $h(k)$ satisfies

$$
0<h_{1} \leq h(k) \leq h_{2}
$$

where $h_{1}$ and $h_{2}$ are known positive integers. Moreover, there exists a constant scalar $w$ such that the function $\delta(\cdot)$ satisfies

$$
\sum_{i=1}^{\infty} \delta(i)=w<\infty
$$

Definition 2.1 ([25]) System (1) is said to be robustly stable if there exists a positivedefinite function $V(k, \xi(k)): Z^{+} \times R^{n} \rightarrow R$ such that

$$
\Delta V(k, \xi(k))=V(k+1, \xi(k+1))-V(k, \xi(k))<0
$$

along any trajectory of solution of system (1) with (2).

Lemma 2.2 ([5]) For any constant matrix $W \in R^{n \times n}, W=W^{T}>0$, two integers $r_{M}$ and $r_{m}$ satisfying $r_{M} \geq r_{m}$, vector function $\xi:\left[r_{m}, r_{M}\right] \rightarrow R^{n}$, the following inequality holds:

$$
\left(\sum_{i=r_{m}}^{r_{M}} \xi(i)\right)^{T} W\left(\sum_{i=r_{m}}^{r_{M}} \xi(i)\right) \leq\left(r_{M}-r_{m}+1\right) \sum_{i=r_{m}}^{r_{M}} \xi^{T}(i) W \xi(i)
$$

Lemma 2.3 ([26]) Let $Q \in R^{n \times n}$ be a positive-definite matrix, $\xi_{i} \in R^{n}, i=1,2, \ldots$. If the sums concerned are well defined, then

$$
\left[\sum_{i=k-M_{1}}^{k-M_{2}-1} \sum_{j=i}^{k-M_{2}-1} \xi_{j}\right]^{T} Q\left[\sum_{i=k-M_{1}}^{k-M_{2}-1} \sum_{j=i}^{k-M_{2}-1} \xi_{j}\right] \leq \frac{\left(M_{1}-M_{2}\right)^{2}}{2} \sum_{i=k-M_{1}}^{k-M_{2}-1} \sum_{j=i}^{k-M_{2}-1} \xi_{j}^{T} Q \xi_{j} .
$$

Lemma 2.4 ([30]) Let $M \in R^{n \times n}$ be a positive-definite matrix, $\xi_{i} \in R^{n}$ and $a_{i} \geq 0, i=$ $1,2, \ldots$ If the sums concerned are well defined, then

$$
\begin{aligned}
& {\left[\sum_{i=k-M_{2}}^{k-1} \xi_{i}\right]^{T} M\left[\sum_{i=k-M_{2}}^{k-1} \xi_{i}\right] \leq M_{2} \sum_{i=k-M_{2}}^{k-1} \xi_{i}^{T} M \xi_{i},} \\
& {\left[\sum_{i=-M_{2}}^{-1} \sum_{i=k+j}^{k-1} \xi_{j}\right]^{T} M\left[\sum_{i=-M_{2}}^{-1} \sum_{i=k+j}^{k-1} \xi_{j}\right] \leq \frac{\left(M_{2}\right)^{2}}{2} \sum_{i=-M_{2}}^{-1} \sum_{i=j+k}^{k-1} \xi_{j}^{T} M \xi_{j},} \\
& {\left[\sum_{i=1}^{\infty} a_{i} \xi_{i}\right]^{T} M\left[\sum_{i=1}^{\infty} a_{i} \xi_{i}\right] \leq\left[\sum_{i=1}^{\infty} a_{i}\right] \sum_{i=1}^{\infty} a_{i} \xi_{i}^{T} M \xi_{i} .}
\end{aligned}
$$




\section{Main results}

Referring to system (1), a new delay-range-dependent robust stability approach is presented here. The Lyapunov-Krasovskii functional method is combined with the LMI technique. Let us introduce the following notations to be used later on:

$$
\begin{aligned}
& L_{w}(\varphi)=\sum_{i=1}^{N} \varphi_{i} L_{i}^{w}, \quad M(\varphi)=\sum_{i=1}^{N} \varphi_{i} M_{i}, \quad Z_{1}(\varphi)=\sum_{i=1}^{N} \varphi_{i} Z_{i}^{1}, \\
& Z_{2}(\varphi)=\sum_{i=1}^{N} \varphi_{i} Z_{i}^{2}, \quad P_{k}(\varphi)=\sum_{i=1}^{N} \varphi_{i} P_{i}^{k}, \quad Q_{s}(\varphi)=\sum_{i=1}^{N} \varphi_{i} Q_{i}^{s}, \\
& M_{r}(\varphi)=\sum_{i=1}^{N} \varphi_{i} M_{i}^{r}, \quad N_{r}(\varphi)=\sum_{i=1}^{N} \varphi_{i} N_{i}^{r}, \\
& \sum_{i=1}^{N} \varphi_{i}=1, \quad \varphi_{i} \geq 0, J, W, L_{i}^{w}, M_{i}, M_{i}^{r}, N_{i}^{r}, Z_{i}^{1}, Z_{i}^{2}, P_{i}^{k}, Q_{i}^{s} \in R^{n \times n}, \\
& w=1,2,3, r=1,2,3,4, k=1,2, \ldots, 18, s=1,2, \ldots, 19, i=1,2, \ldots, N, \\
& \prod_{i, j}=\left[\Sigma_{i, j}^{m, n}\right]_{27 \times 27},
\end{aligned}
$$

where $\Sigma_{i, j}^{m, n}=\Sigma_{i, j}^{n, m T}, m, n=1,2,3, \ldots, 27, i, j=1,2,3, \ldots, N$,

$$
\begin{aligned}
& \Sigma_{i, j}^{1,1}=G_{i}^{1}+G_{i}^{2}+G_{i}^{1 T}+G_{i}^{2^{T}}-L_{i}^{1^{T}} A_{j}^{1}+L_{i}^{1^{T}} B_{j}^{1}-L_{i}^{1^{T}}+A_{i}^{1^{T}} L_{j}^{1} \\
& +B_{i}^{1^{T}} L_{j}^{1}-L_{i}^{1}+P_{i}^{2}+P_{i}^{3}+P_{i}^{4}+P_{i}^{5}+P_{i}^{8}+P_{i}^{9}+h_{1}^{2} Q_{i}^{1} \\
& +r^{2} h_{1}^{2} Q_{i}^{4}+h_{2}^{2} Q_{i}^{7}+r^{2} h_{2}^{2} Q_{i}^{10}+\rho^{2} Q_{i}^{13}+r^{2} \rho^{2} Q_{i}^{16}+h_{1} M_{i}^{1} \\
& +N_{i}^{1}+N_{i}^{1 T}+h_{2} M_{i}^{2}+N_{i}^{2}+N_{i}^{2^{T}}+r h_{1} M_{i}^{3}+N_{i}^{3}+N_{i}^{3 T} \\
& +r h_{2} M_{i}^{4}+N_{i}^{4}+N_{i}^{4 T}-h_{1}^{2} P_{i}^{17}+\epsilon_{1} \varphi^{2} I+w P_{i}^{19}, \\
& \Sigma_{i, j}^{1,2}=P_{i}^{1}+G_{i}^{1^{T}}+G_{i}^{2^{T}}-L_{i}^{1^{T}}+A_{i}^{1^{T}} L_{j}^{2}+B_{i}^{1^{T}} L_{j}^{2}-L_{i}^{2}+h_{1}^{2} Q_{i}^{2} \\
& +r^{2} h_{1}^{2} Q_{i}^{5}+h_{2}^{2} Q_{i}^{8}+r^{2} h_{2}^{2} Q_{i}^{11}+\rho^{2} Q_{i}^{14}+r^{2} \rho^{2} Q_{i}^{17}, \\
& \Sigma_{i, j}^{1,3}=-N_{i}^{1}, \quad \Sigma_{i, j}^{1,4}=-N_{i}^{2}, \quad \Sigma_{i, j}^{1,5}=-G_{i}^{1}+L_{i}^{1^{T}} B_{j}^{2}, \quad \Sigma_{i, j}^{1,6}=-N_{i}^{3}, \\
& \Sigma_{i, j}^{1,7}=-N_{i}^{4}, \quad \Sigma_{i, j}^{1,8}=-G_{i}^{2}+L_{i}^{1^{T}} A_{j}^{2}+h_{1} P_{i}^{17}, \quad \Sigma_{i, j}^{1,21}=-G_{i}^{1}-L_{i}^{1^{T}} B_{j}^{1}, \\
& \Sigma_{i, j}^{1,22}=-G_{i}^{2}+L_{i}^{1^{T}} A_{j}^{2}+A_{i}^{1^{T}} L_{j}^{3}+B_{i}^{1^{T}} L_{j}^{3}-L_{i}^{3}, \\
& \Sigma_{i, j}^{1,25}=L_{i}^{1^{T}}, \quad \Sigma_{i, j}^{1,26}=L_{i}^{1^{T}}, \quad \Sigma_{i, j}^{1,27}=L_{i}^{1^{T}} C_{j}, \\
& \Sigma_{i, j}^{2,2}=P_{i}^{1}-L_{i}^{2 T}-L_{i}^{2}+h_{1}^{2} Q_{i}^{3}+r^{2} h_{1}^{2} Q_{i}^{6}+h_{2}^{2} Q_{i}^{9}+r^{2} h_{2}^{2} Q_{i}^{12}+\rho^{2} Q_{i}^{15} \\
& +r^{2} \rho^{2} Q_{i}^{18}+h_{1} P_{i}^{10}+h_{2} P_{i}^{11}+r h_{1} P_{i}^{12}+r h_{2} P_{i}^{13}+h_{1}^{2} P_{i}^{14} \\
& +h_{2}^{2} P_{i}^{15}+\rho^{2} P_{i}^{16}+\frac{h_{1}^{4}}{4} P_{i}^{17}+\frac{\left(h_{2}-h_{1}\right)^{2}}{4} P_{i}^{18}+h_{2}^{2} Z_{i}^{1}+\left(r h_{2}\right)^{2} Z_{i}^{2}, \\
& \Sigma_{i, j}^{2,5}=-G_{i}^{1}+L_{i}^{2^{T}} B_{j}^{2}, \quad \Sigma_{i, j}^{2,8}=-G_{i}^{2}+L_{i}^{2^{T}} A_{j}^{2}, \quad \Sigma_{i, j}^{2,21}=-G_{i}^{1}+L_{i}^{2^{T}} B_{j}^{1}, \\
& \Sigma_{i, j}^{2,22}=-G_{i}^{2}+L_{i}^{2^{T}} A_{j}^{2}-L_{i}^{3}, \quad \Sigma_{i, j}^{2,25}=L_{i}^{2^{T}}, \quad \Sigma_{i, j}^{2,26}=L_{i}^{2^{T}},
\end{aligned}
$$




$$
\begin{aligned}
& \Sigma_{i, j}^{2,27}=L_{i}^{2^{T}} C_{j}, \quad \Sigma_{i, j}^{3,3}=-P_{i}^{2}+P_{i}^{6}-P_{i}^{18}, \quad \Sigma_{i, j}^{3,23}=P_{i}^{18}, \\
& \Sigma_{i, j}^{4,4}=-P_{i}^{4}-P_{i}^{6}, \quad \Sigma_{i, j}^{5,5}=-P_{i}^{8}-P_{i}^{18}+\epsilon_{2} \beta^{2} I, \quad \Sigma_{i, j}^{5,22}=B_{i}^{2^{T}} L_{j}^{3}, \\
& \Sigma_{i, j}^{5,24}=P_{i}^{18}, \quad \Sigma_{i, j}^{6,6}=-P_{i}^{3}+P_{i}^{7}, \quad \Sigma_{i, j}^{7,7}=-P_{i}^{5}-P_{i}^{7}, \\
& \Sigma_{i, j}^{8,8}=-P_{i}^{9}-P_{i}^{17}, \quad \Sigma_{i, j}^{8,22}=A_{i}^{2^{T}} L_{j}^{3}, \quad \Sigma_{i, j}^{9,9}=-Q_{i}^{1}, \Sigma_{i, j}^{9,15}=-Q_{i}^{2}, \\
& \Sigma_{i, j}^{10,10}=-Q_{i}^{4}, \quad \Sigma_{i, j}^{10,16}=-Q_{i}^{5}, \quad \Sigma_{i, j}^{11,11}=-Q_{i}^{7}, \quad \Sigma_{i, j}^{11,17}=-Q_{i}^{8}, \\
& \Sigma_{i, j}^{12,12}=-Q_{i}^{10}, \quad \Sigma_{i, j}^{12,18}=-Q_{i}^{11}, \quad \Sigma_{i, j}^{13,13}=-Q_{i}^{13}, \quad \Sigma_{i, j}^{13,19}=-Q_{i}^{14}, \\
& \Sigma_{i, j}^{14,14}=-Q_{i}^{16}, \quad \Sigma_{i, j}^{14,20}=-Q_{i}^{17}, \quad \Sigma_{i, j}^{15,15}=-Q_{i}^{3}-P_{i}^{14}, \quad \Sigma_{i, j}^{16,16}=-Q_{i}^{6}, \\
& \Sigma_{i, j}^{17,17}=-Q_{i}^{9}-P_{i}^{15}, \quad \Sigma_{i, j}^{18,18}=-Q_{i}^{12}, \quad \Sigma_{i, j}^{19,19}=-Q_{i}^{15}-P_{i}^{16}, \\
& \Sigma_{i, j}^{21,21}=-Z_{i}^{1}, \quad \Sigma_{i, j}^{21,22}=-B_{i}^{1^{T}} L_{j}^{3}, \quad \Sigma_{i, j}^{21,25}=L_{i}^{5^{T}}, \quad \Sigma_{i, j}^{21,26}=L_{i}^{5^{T}}, \\
& \Sigma_{i, j}^{21,27}=L_{i}^{5^{T}} C_{j}, \quad \Sigma_{i, j}^{22,22}=L_{i}^{3^{T}} A_{j}^{2}+A_{i}^{2^{T}} L_{j}^{3}-Z_{i}^{2}, \quad \Sigma_{i, j}^{22,25}=L_{i}^{3^{T}}, \\
& \Sigma_{i, j}^{22,26}=L_{i}^{3^{T}}, \quad \Sigma_{i, j}^{22,27}=L_{i}^{3^{T}} C_{j}, \quad \Sigma_{i, j}^{23,23}=-P_{i}^{18}, \quad \Sigma_{i, j}^{24,24}=-P_{i}^{18}, \\
& \Sigma_{i, j}^{25,25}=-\epsilon_{1} I, \quad \Sigma_{i, j}^{26,26}=-\epsilon_{2} I, \quad \Sigma_{i, j}^{27,27}=-\frac{P_{i}^{19}}{w}, \quad G_{i}^{1}=P_{i}^{1} J, \\
& G_{i}^{2}=P_{i}^{1} W, \quad \rho=h_{2}-h_{1}, \quad \varphi(k)=h(k)-h_{1}, \quad \beta(k)=h_{2}-h(k), \\
& \psi(k)=\frac{1}{\varphi(k)}\left[\sum_{i=k-h(k)}^{k-h_{1}-1} \xi(i)\right], \quad \phi(k)=\frac{1}{\beta(k)}\left[\sum_{i=k-h_{2}}^{k-h(k)-1} \xi(i)\right],
\end{aligned}
$$

and other terms are 0 .

Theorem 3.1 System (1) is robustly stable if there exist positive definite symmetric matrices $M_{i}, Z_{i}^{1}, Z_{i}^{2}, P_{i}^{k}, Q_{i}^{s}(k=1,2, \ldots, 19, s=1,2, \ldots, 18, i=1,2, \ldots, N)$, any matrices $J, W, M_{i}^{r}, N_{i}^{r}, L_{i}^{l}(r=1,2,3,4, l=1,2,3, i=1,2, \ldots, N)$ of appropriate dimensions, and positive real constants $\epsilon_{1}$ and $\epsilon_{2}$ satisfying the following LMIs:

$$
\begin{aligned}
& \prod_{i, i}<-I, \quad i=1,2, \ldots, N, \\
& \prod_{i, j}+\prod_{j, i}<\frac{2}{(N-1)} I, \quad i=1, \ldots, N-1, j=i+1, \ldots, N, \\
& {\left[\begin{array}{cc}
Q_{i}^{3 t+1} & Q_{i}^{3 t+2} \\
* & Q_{i}^{3 t+3}
\end{array}\right] \geq 0, \quad t=0,1,2, \ldots, 5, i=1,2, \ldots, N,} \\
& {\left[\begin{array}{cc}
M_{i}^{l} & N_{i}^{l} \\
* & P_{i}^{9+l}
\end{array}\right] \geq 0, \quad l=1,2, \ldots, 4, i=1,2, \ldots, N .}
\end{aligned}
$$

Proof Applying a model transformation method, system (1) can be replaced by the system

$$
\begin{aligned}
& \xi(k+1)=\xi(k)+\eta(k), \\
& \eta(k)=[A(\varphi)-I] \xi(k)+B(\varphi) \xi(k-h(k))+C(\varphi) \sum_{i=1}^{\infty} \delta(i) \xi(k-i)
\end{aligned}
$$




$$
+f_{1}(k, \xi(k))+f_{2}(k, \xi(k-h(k)))
$$

To improve the bound for $h(k)$ in (1), we decompose constant matrices $A$ and $B$ as

$$
\begin{aligned}
& A(\varphi)=A_{1}(\varphi)+A_{2}(\varphi), \\
& B(\varphi)=B_{1}(\varphi)+B_{2}(\varphi),
\end{aligned}
$$

where $A_{1}(\varphi)=\sum_{i=1}^{N} \varphi_{i} A_{i}^{1}, A_{2}(\varphi)=\sum_{i=1}^{N} \varphi_{i} A_{i}^{2}, B_{1}(\varphi)=\sum_{i=1}^{N} \varphi_{i} B_{i}^{1}, B_{2}(\varphi)=\sum_{i=1}^{N} \varphi_{i} B_{i}^{2}$, and $A_{i}^{1}, A_{i}^{2}, B_{i}^{1}, B_{i}^{2} \in R^{n \times n}$ are constant matrices for all $i=1,2,3, \ldots, N$. By utilizing the following zero equations, we have

$$
\begin{aligned}
& \xi(k)-\xi(k-h(k))-\sum_{i=k-h(k)}^{k-1} \eta(i)=0, \\
& \xi(k)-\xi(k-r h(k))-\sum_{i=k-r h(k)}^{k-1} \eta(i)=0 .
\end{aligned}
$$

From (15) and (16), we obtain

$$
\begin{aligned}
& J \xi(k)-J \xi(k-h(k))-J \sum_{i=k-h(k)}^{k-1} \eta(i)=0, \\
& W \xi(k)-W \xi(k-r h(k))-W \sum_{i=k-r h(k)}^{k-1} \eta(i)=0,
\end{aligned}
$$

where $J, W \in R^{n \times n}$ are selected so that the stability criterion of the given system is guaranteed. Substituting (17)-(18) into the system (11)-(12), we obtain

$$
\begin{aligned}
\xi(k+1)= & \xi(k)+\eta(k)+J \xi(k)-J \xi(k-h(k))-J \sum_{i=k-h(k)}^{k-1} \eta(i) \\
& +W \xi(k)-W \xi(k-r h(k))-W \sum_{i=k-r h(k)}^{k-1} \eta(i), \\
\eta(k)=\left[A_{1}(\varphi)+B_{1}(\varphi)-I\right] \xi(k)+A_{2}(\varphi) \xi(k-r h(k)) & \\
+ & B_{2}(\varphi) \xi(k-h(k))-B_{1}(\varphi) \sum_{i=k-h(k)}^{k-1} \eta(i)+A_{2}(\varphi) \sum_{i=k-r h(k)}^{k-1} \eta(i) \\
& +C(\varphi) \sum_{i=1}^{\infty} \delta(i) \xi(k-i)+f_{1}(k, \xi(k))+f_{2}(k, \xi(k-h(k))) .
\end{aligned}
$$

We define a parameter-dependent Lyapunov-Krasovskii functional for the system (19)(20) as

$$
V(k, \xi(k))=\sum_{i=1}^{8} V_{i}(k, \xi(k))
$$


where

$$
\begin{aligned}
& V_{1}(k, \xi(k))=\xi^{T}(k) P_{1}(\varphi) \xi(k), \\
& V_{2}(k, \xi(k))=\sum_{i=k-h_{1}}^{k-1} \xi^{T}(i) P_{2}(\varphi) \xi(i)+\sum_{i=k-r h_{1}}^{k-1} \xi^{T}(i) P_{3}(\varphi) \xi(i) \\
& +\sum_{i=k-h_{2}}^{k-1} \xi^{T}(i) P_{4}(\varphi) \xi(i)+\sum_{i=k-r h_{2}}^{k-1} \xi^{T}(i) P_{5}(\varphi) \xi(i) \\
& +\sum_{i=k-h_{2}}^{k-h_{1}-1} \xi^{T}(i) P_{6}(\varphi) \xi(i)+\sum_{i=k-r h_{2}}^{k-r h_{1}-1} \xi^{T}(i) P_{7}(\varphi) \xi(i) \\
& +\sum_{i=k-h(k)}^{k-1} \xi^{T}(i) P_{8}(\varphi) \xi(i)+\sum_{i=k-r h(k)}^{k-1} \xi^{T}(i) P_{9}(\varphi) \xi(i) \\
& V_{3}(k, \xi(k))=h_{1} \sum_{j=-h_{1}}^{-1} \sum_{i=k+j}^{k-1}\left[\begin{array}{c}
\xi(i) \\
\eta(i)
\end{array}\right]^{T}\left[\begin{array}{cc}
Q_{1}(\varphi) & Q_{2}(\varphi) \\
* & Q_{3}(\varphi)
\end{array}\right]\left[\begin{array}{c}
\xi(i) \\
\eta(i)
\end{array}\right] \\
& +r h_{1} \sum_{j=-r h_{1}}^{-1} \sum_{i=k+j}^{k-1}\left[\begin{array}{l}
\xi(i) \\
\eta(i)
\end{array}\right]^{T}\left[\begin{array}{cc}
Q_{4}(\varphi) & Q_{5}(\varphi) \\
* & Q_{6}(\varphi)
\end{array}\right]\left[\begin{array}{l}
\xi(i) \\
\eta(i)
\end{array}\right] \\
& +h_{2} \sum_{j=-h_{2}}^{-1} \sum_{i=k+j}^{k-1}\left[\begin{array}{c}
\xi(i) \\
\eta(i)
\end{array}\right]^{T}\left[\begin{array}{cc}
Q_{7}(\varphi) & Q_{8}(\varphi) \\
* & Q_{9}(\varphi)
\end{array}\right]\left[\begin{array}{c}
\xi(i) \\
\eta(i)
\end{array}\right] \\
& +r h_{2} \sum_{j=-r h_{2}}^{-1} \sum_{i=k+j}^{k-1}\left[\begin{array}{c}
\xi(i) \\
\eta(i)
\end{array}\right]^{T}\left[\begin{array}{cc}
Q_{10}(\varphi) & Q_{11}(\varphi) \\
* & Q_{12}(\varphi)
\end{array}\right]\left[\begin{array}{c}
\xi(i) \\
\eta(i)
\end{array}\right] \\
& +\rho \sum_{j=-h_{2}}^{-h_{1}-1} \sum_{i=k+j}^{k-1}\left[\begin{array}{c}
\xi(i) \\
\eta(i)
\end{array}\right]^{T}\left[\begin{array}{cc}
Q_{13}(\varphi) & Q_{14}(\varphi) \\
* & Q_{15}(\varphi)
\end{array}\right]\left[\begin{array}{l}
\xi(i) \\
\eta(i)
\end{array}\right] \\
& +r \rho \sum_{j=-r h_{2}}^{-r h_{1}-1} \sum_{i=k+j}^{k-1}\left[\begin{array}{l}
\xi(i) \\
\eta(i)
\end{array}\right]^{T}\left[\begin{array}{cc}
Q_{16}(\varphi) & Q_{17}(\varphi) \\
* & Q_{18}(\varphi)
\end{array}\right]\left[\begin{array}{l}
\xi(i) \\
\eta(i)
\end{array}\right], \\
& V_{4}(k, \xi(k))=\sum_{j=-h_{1}+1}^{0} \sum_{i=k-1+j}^{k-1} \eta^{T}(i) P_{10}(\varphi) \eta(i) \\
& +\sum_{j=-h_{2}+1}^{0} \sum_{i=k-1+j}^{k-1} \eta^{T}(i) P_{11}(\varphi) \eta(i) \\
& +\sum_{j=-r h_{1}+1}^{0} \sum_{i=k-1+j}^{k-1} \eta^{T}(i) P_{12}(\varphi) \eta(i) \\
& +\sum_{j=-r h_{2}+1}^{0} \sum_{i=k-1+j}^{k-1} \eta^{T}(i) P_{13}(\varphi) \eta(i) \\
& +\sum_{i=1}^{\infty} \delta(i) \sum_{j=k-i}^{k-1} \xi_{j}^{T} M(\varphi) \xi_{j}
\end{aligned}
$$




$$
\begin{aligned}
V_{5}(k, \xi(k))= & h_{1} \sum_{j=-h_{1}}^{-1} \sum_{i=k+j}^{k-1} \eta^{T}(i) P_{14}(\varphi) \eta(i) \\
& +h_{2} \sum_{j=-h_{2}}^{-1} \sum_{i=k+j}^{k-1} \eta^{T}(i) P_{15}(\varphi) \eta(i) \\
& +\rho \sum_{j=-h_{2}}^{-h_{1}-1} \sum_{i=k+j}^{k-1} \eta^{T}(i) P_{16}(\varphi) \eta(i), \\
V_{6}(k, \xi(k))= & h_{2} \sum_{j=-h_{2}}^{-1} \sum_{i=k+j}^{k-1} \eta^{T}(i) Z_{1}(\varphi) \eta(i) \\
& +r h_{2} \sum_{j=-r h_{2}}^{-1} \sum_{i=k+j}^{k-1} \eta^{T}(i) Z_{2}(\varphi) \eta(i), \\
V_{7}(k, \xi(k))= & \frac{h_{1}^{2}}{2} \sum_{i=-h_{1}}^{-1} \sum_{j=i}^{0} \sum_{l=k+j}^{k-1} \eta^{T}(l) P_{17}(\varphi) \eta(l) \\
& +\frac{1}{2} \sum_{i=k-h_{2}}^{k-h_{1}-1} \sum_{j=i}^{k-h_{1}-1} \sum_{l=j}^{k-h_{1}-1} \eta^{T}(l) P_{18}(\varphi) \eta(l), \\
V_{8}(k, \xi(k))= & \sum_{i=1}^{\infty} \delta(i) \sum_{j=k-i}^{k-1} \xi^{T}(j) P_{19}(\varphi) \xi(j) .
\end{aligned}
$$

From (21), the forward difference of $V(k, \xi(k))$ is defined by

$$
\Delta V(k, \xi(k))=\sum_{i=1}^{8} \Delta V_{i}(k, \xi(k))
$$

where

$$
\Delta V_{i}(k, \xi(k))=V_{i}(k+1, \xi(k+1))-V_{i}(k, \xi(k))
$$

for $i=1,2, \ldots, 8$. The increments of $V_{1}(k, \xi(k)), V_{2}(k, \xi(k))$, and $V_{3}(k, \xi(k))$ are obtained by taking the forward difference of $V_{1}(k, \xi(k)), V_{2}(k, \xi(k))$, and $V_{3}(k, \xi(k))$, respectively. Then, we have

$$
\begin{aligned}
\Delta V_{1}(k, \xi(k))= & \xi^{T}(k+1) P_{1}(\varphi) \xi(k+1)-\xi^{T}(k) P_{1}(\varphi) \xi(k) \\
= & {\left[\xi^{T}(k)+\eta^{T}(k)\right] P_{1}(\varphi)[\xi(k)+\eta(k)+J \xi(k)} \\
& -J \xi(k-h(k))-J \sum_{i=k-h(k)}^{k-1} \eta(i)+W \xi(k) \\
& \left.-W \xi(k-r h(k))-W \sum_{i=k-r h(k)}^{k-1} \eta(i)\right]
\end{aligned}
$$




$$
\begin{aligned}
& +\left[\xi^{T}(k) J^{T}-\xi^{T}(k-h(k)) J^{T}\right. \\
& -\sum_{i=k-h(k)}^{k-1} \eta^{T}(i) J^{T}+\xi^{T}(k) W^{T}-\xi^{T}(k-r h(k)) W^{T} \\
& \left.-\sum_{i=k-r h(k)}^{k-1} \eta^{T}(i) W^{T}\right] P_{1}(\varphi)[\xi(k)+\eta(k)] \\
& +2 \xi^{T}(k) L_{1}^{T}(\varphi)\left[-\eta(k)+\left[A_{1}(\varphi)+B_{1}(\varphi)-I\right] \xi(k)\right. \\
& +A_{2}(\varphi) \xi(k-r h(k))+B_{2}(\varphi) \xi(k-h(k)) \\
& -B_{1}(\varphi) \sum_{i=k-h(k)}^{k-1} \eta(i)+A_{2}(\varphi) \sum_{i=k-r h(k)}^{k-1} \eta(i) \\
& \left.+C(\varphi) \sum_{i=1}^{\infty} \delta(i) \xi(k-i)+f_{1}(k, \xi(k))+f_{2}(k, \xi(k-h(k)))\right] \\
& +2 \eta^{T}(k) L_{2}^{T}(\varphi)\left[-\eta(k)+\left[A_{1}(\varphi)+B_{1}(\varphi)-I\right] \xi(k)\right. \\
& +A_{2}(\varphi) \xi(k-r h(k))+B_{2}(\varphi) \xi(k-h(k)) \\
& -B_{1}(\varphi) \sum_{i=k-h(k)}^{k-1} \eta(i)+A_{2}(\varphi) \sum_{i=k-r h(k)}^{k-1} \eta(i) \\
& \left.+C(\varphi) \sum_{i=1}^{\infty} \delta(i) \xi(k-i)+f_{1}(k, \xi(k))+f_{2}(k, \xi(k-h(k)))\right] \\
& +2 \sum_{i=k-r h(k)}^{k-1} \eta^{T}(i) L_{3}^{T}(\varphi)\left[-\eta(k)+\left[A_{1}(\varphi)+B_{1}(\varphi)-I\right] \xi(k)\right. \\
& +A_{2}(\varphi) \xi(k-r h(k))+B_{2}(\varphi) \xi(k-h(k)) \\
& -B_{1}(\varphi) \sum_{i=k-h(k)}^{k-1} \eta(i)+A_{2}(\varphi) \sum_{i=k-r h(k)}^{k-1} \eta(i) \\
& \left.+C(\varphi) \sum_{i=1}^{\infty} \delta(i) \xi(k-i)+f_{1}(k, \xi(k))+f_{2}(k, \xi(k-h(k)))\right] \\
& -\xi^{T}(k) P_{1}(\varphi) \xi(k), \\
& \Delta V_{2}(k, \xi(k))=\xi^{T}(k) P_{2}(\varphi) \xi(k)-\xi^{T}\left(k-h_{1}\right) P_{2}(\varphi) \xi\left(k-h_{1}\right) \\
& +\xi^{T}(k) P_{3}(\varphi) \xi(k)-\xi^{T}\left(k-r h_{1}\right) P_{3}(\varphi) \xi\left(k-r h_{1}\right) \\
& +\xi^{T}(k) P_{4}(\varphi) \xi(k)-\xi^{T}\left(k-h_{2}\right) P_{4}(\varphi) \xi\left(k-h_{2}\right) \\
& +\xi^{T}(k) P_{5}(\varphi) \xi(k)-\xi^{T}\left(k-r h_{2}\right) P_{5}(\varphi) \xi\left(k-r h_{2}\right) \\
& +\xi^{T}\left(k-h_{1}\right) P_{6}(\varphi) \xi\left(k-h_{1}\right)-\xi^{T}\left(k-h_{2}\right) P_{6}(\varphi) \xi\left(k-h_{2}\right) \\
& +\xi^{T}\left(k-r h_{1}\right) P_{7}(\varphi) \xi\left(k-r h_{1}\right)
\end{aligned}
$$




$$
\begin{aligned}
& -\xi^{T}\left(k-r h_{2}\right) P_{7}(\varphi) \xi\left(k-r h_{2}\right) \\
& +\xi^{T}(k) P_{8}(\varphi) \xi(k)-\xi^{T}(k-h(k)) P_{8}(\varphi) \xi(k-h(k)) \\
& +\xi^{T}(k) P_{9}(\varphi) \xi(k)-\xi^{T}(k-r h(k)) P_{9}(\varphi) \xi(k-r h(k)), \\
& \Delta V_{3}(k, \xi(k)) \leq\left[\begin{array}{c}
\xi(k) \\
\eta(k)
\end{array}\right]^{T}\left[\begin{array}{cc}
h_{1}^{2} Q_{1}(\varphi) & h_{1}^{2} Q_{2}(\varphi) \\
* & h_{1}^{2} Q_{3}(\varphi)
\end{array}\right]\left[\begin{array}{c}
\xi(k) \\
\eta(k)
\end{array}\right] \\
& -\left[\begin{array}{c}
\sum_{i=k-h_{1}}^{k-1} \xi(i) \\
\sum_{i=k-h_{1}}^{k-1} \eta(i)
\end{array}\right]^{T}\left[\begin{array}{cc}
Q_{1}(\varphi) & Q_{2}(\varphi) \\
* & Q_{3}(\varphi)
\end{array}\right]\left[\begin{array}{c}
\sum_{i=k-h_{1}}^{k-1} \xi(i) \\
\sum_{i=k-h_{1}}^{k-1} \eta(i)
\end{array}\right] \\
& +\left[\begin{array}{c}
\xi(k) \\
\eta(k)
\end{array}\right]^{T}\left[\begin{array}{cc}
r^{2} h_{1}^{2} Q_{4}(\varphi) & r^{2} h_{1}^{2} Q_{5}(\varphi) \\
* & r^{2} h_{1}^{2} Q_{6}(\varphi)
\end{array}\right]\left[\begin{array}{c}
\xi(k) \\
\eta(k)
\end{array}\right] \\
& -\left[\begin{array}{c}
\sum_{i=k-r h_{1}}^{k-1} \xi(i) \\
\sum_{i=k-r h_{1}}^{k-1} \eta(i)
\end{array}\right]^{T}\left[\begin{array}{cc}
Q_{4}(\varphi) & Q_{5}(\varphi) \\
* & Q_{6}(\varphi)
\end{array}\right]\left[\begin{array}{c}
\sum_{i=k-r h_{1}}^{k-1} \xi(i) \\
\sum_{i=k-r h_{1}}^{k-1} \eta(i)
\end{array}\right] \\
& +\left[\begin{array}{c}
\xi(k) \\
\eta(k)
\end{array}\right]^{T}\left[\begin{array}{cc}
h_{2}^{2} Q_{7}(\varphi) & h_{2}^{2} Q_{8}(\varphi) \\
* & h_{2}^{2} Q_{9}(\varphi)
\end{array}\right]\left[\begin{array}{c}
\xi(k) \\
\eta(k)
\end{array}\right] \\
& \text { - }\left[\begin{array}{c}
\sum_{i=k-h_{2}}^{k-1} \xi(i) \\
\sum_{i=k-h_{2}}^{k-1} \eta(i)
\end{array}\right]^{T}\left[\begin{array}{cc}
Q_{7}(\varphi) & Q_{8}(\varphi) \\
* & Q_{9}(\varphi)
\end{array}\right]\left[\begin{array}{c}
\sum_{i=k-h_{2}}^{k-1} \xi(i) \\
\sum_{i=k-h_{2}}^{k-1} \eta(i)
\end{array}\right] \\
& +\left[\begin{array}{c}
\xi(k) \\
\eta(k)
\end{array}\right]^{T}\left[\begin{array}{cc}
r^{2} h_{2}^{2} Q_{10}(\varphi) & r^{2} h_{2}^{2} Q_{11}(\varphi) \\
* & r^{2} h_{2}^{2} Q_{12}(\varphi)
\end{array}\right]\left[\begin{array}{c}
\xi(k) \\
\eta(k)
\end{array}\right] \\
& -\left[\begin{array}{c}
\sum_{i=k-r h_{2}}^{k-1} \xi(i) \\
\sum_{i=k-r h_{2}}^{k-1} \eta(i)
\end{array}\right]^{T}\left[\begin{array}{cc}
Q_{10}(\varphi) & Q_{11}(\varphi) \\
* & Q_{12}(\varphi)
\end{array}\right]\left[\begin{array}{c}
\sum_{i=k-r h_{2}}^{k-1} \xi(i) \\
\sum_{i=k-r h_{2}}^{k-1} \eta(i)
\end{array}\right] \\
& +\left[\begin{array}{c}
\xi(k) \\
\eta(k)
\end{array}\right]^{T}\left[\begin{array}{cc}
\rho^{2} Q_{13}(\varphi) & \rho^{2} Q_{14}(\varphi) \\
* & \rho^{2} Q_{15}(\varphi)
\end{array}\right]\left[\begin{array}{l}
\xi(k) \\
\eta(k)
\end{array}\right] \\
& -\left[\begin{array}{c}
\sum_{i=k-1}^{k-h_{1}-1} \xi(i) \\
\sum_{i=k-h_{2}}^{k-h_{1}-1} \eta(i)
\end{array}\right]^{T}\left[\begin{array}{cc}
Q_{13}(\varphi) & Q_{14}(\varphi) \\
* & Q_{15}(\varphi)
\end{array}\right]\left[\begin{array}{c}
\sum_{i=k-1}^{k-h_{1}-1} \xi(i) \\
\sum_{i=k-h_{2}}^{k-h_{2}-1} \eta(i)
\end{array}\right] \\
& +\left[\begin{array}{c}
\xi(k) \\
\eta(k)
\end{array}\right]^{T}\left[\begin{array}{cc}
r^{2} \rho^{2} Q_{16}(\varphi) & r^{2} \rho^{2} Q_{17}(\varphi) \\
* & r^{2} \rho^{2} Q_{18}(\varphi)
\end{array}\right]\left[\begin{array}{c}
\xi(k) \\
\eta(k)
\end{array}\right] \\
& -\left[\begin{array}{c}
\sum_{i=k-k-r h_{2}-1}^{k-r} \xi(i) \\
\sum_{i=k-r h_{2}}^{k-r h_{1}-1} \eta(i)
\end{array}\right]^{T}\left[\begin{array}{cc}
Q_{16}(\varphi) & Q_{17}(\varphi) \\
* & Q_{18}(\varphi)
\end{array}\right]\left[\begin{array}{c}
\sum_{i=k-r h_{1}}^{k-r h_{1}-1} \xi(i) \\
\sum_{i=k-r h_{2}}^{k-r h_{1}-1} \eta(i)
\end{array}\right] .
\end{aligned}
$$

Taking the forward difference of $V_{4}(k, \xi(k))$ yields

$$
\begin{aligned}
& \Delta V_{4}(k, \xi(k)) \\
& =h_{1} \eta^{T}(k) P_{10}(\varphi) \eta(k)-\sum_{i=-h_{1}+1}^{0} \eta^{T}(k-1+i) P_{10}(\varphi) \eta(k-1+i) \\
& \quad+h_{2} \eta^{T}(k) P_{11}(\varphi) \eta(k)-\sum_{i=-h_{2}+1}^{0} \eta^{T}(k-1+i) P_{11}(\varphi)
\end{aligned}
$$




$$
\begin{aligned}
& \times \eta(k-1+i)+r h_{1} \eta^{T}(k) P_{12}(\varphi) \eta(k)-\sum_{i=-r h_{1}+1}^{0} \eta^{T}(k-1+i) \\
& \times P_{12}(\varphi) \eta(k-1+i)+r h_{2} \eta^{T}(k) P_{13}(\varphi) \eta(k) \\
& -\sum_{i=-r h_{2}+1}^{0} \eta^{T}(k-1+i) P_{13}(\varphi) \eta(k-1+i) .
\end{aligned}
$$

By (10), it is easy to see that

$$
\begin{aligned}
& 2 \xi^{T}(k) N_{1}(\varphi) \sum_{i=-h_{1}+1}^{0} \eta(k-1+i)+\sum_{i=-h_{1}+1}^{0} \eta^{T}(k-1+i) P_{10}(\varphi) \eta(k-1+i) \\
& +h_{1} \xi^{T}(k) M_{1}(\varphi) \xi(k) \\
& =\sum_{i=-h_{1}+1}^{0}\left[\begin{array}{c}
\xi(k) \\
\eta(k-1+i)
\end{array}\right]^{T}\left[\begin{array}{cc}
M_{1}(\varphi) & N_{1}(\varphi) \\
* & P_{10}(\varphi)
\end{array}\right]\left[\begin{array}{c}
\xi(k) \\
\eta(k-1+i)
\end{array}\right] \geq 0, \\
& 2 \xi^{T}(k) N_{2}(\varphi) \sum_{i=-h_{2}+1}^{0} \eta(k-1+i)+\sum_{i=-h_{2}+1}^{0} \eta^{T}(k-1+i) P_{11}(\varphi) \eta(k-1+i) \\
& +h_{2} \xi^{T}(k) M_{2}(\varphi) \xi(k) \\
& =\sum_{i=-h_{2}+1}^{0}\left[\begin{array}{c}
\xi(k) \\
\eta(k-1+i)
\end{array}\right]^{T}\left[\begin{array}{cc}
M_{2}(\varphi) & N_{2}(\varphi) \\
* & P_{11}(\varphi)
\end{array}\right]\left[\begin{array}{c}
\xi(k) \\
\eta(k-1+i)
\end{array}\right] \geq 0, \\
& 2 \xi^{T}(k) N_{3}(\varphi) \sum_{i=-r h_{1}+1}^{0} \eta(k-1+i)+\sum_{i=-r h_{1}+1}^{0} \eta^{T}(k-1+i) P_{12}(\varphi) \\
& \times \eta(k-1+i)+r h_{1} \xi^{T}(k) M_{3}(\varphi) \xi(k) \\
& =\sum_{i=-r h_{1}+1}^{0}\left[\begin{array}{c}
\xi(k) \\
\eta(k-1+i)
\end{array}\right]^{T}\left[\begin{array}{cc}
M_{3}(\varphi) & N_{3}(\varphi) \\
* & P_{12}(\varphi)
\end{array}\right]\left[\begin{array}{c}
\xi(k) \\
\eta(k-1+i)
\end{array}\right] \geq 0, \\
& 2 \xi^{T}(k) N_{4}(\varphi) \sum_{i=-r h_{2}+1}^{0} \eta(k-1+i)+\sum_{i=-r h_{2}+1}^{0} \eta^{T}(k-1+i) P_{13}(\varphi) \\
& \times \eta(k-1+i)+r h_{2} \xi^{T}(k) M_{4}(\varphi) \xi(k) \\
& =\sum_{i=-r h_{2}+1}^{0}\left[\begin{array}{c}
\xi(k) \\
\eta(k-1+i)
\end{array}\right]^{T}\left[\begin{array}{cc}
M_{4}(\varphi) & N_{4}(\varphi) \\
* & P_{13}(\varphi)
\end{array}\right]\left[\begin{array}{c}
\xi(k) \\
\eta(k-1+i)
\end{array}\right] \geq 0 .
\end{aligned}
$$

From (28), (29), (30), and (31), we obtain

$$
\begin{aligned}
& -\sum_{i=-h_{1}+1}^{0} \eta^{T}(k-1+i) P_{10}(\varphi) \eta(k-1+i) \\
& \quad \leq h_{1} \xi^{T}(k) M_{1}(\varphi) \xi(k)+2 \xi^{T}(k) N_{1}(\varphi) \sum_{i=-h_{1}+1}^{0} \eta(k-1+i) \\
& =h_{1} \xi^{T}(k) M_{1}(\varphi) \xi(k)+2 \xi^{T}(k) N_{1}(\varphi) \xi(k)-2 \xi^{T}(k) N_{1}(\varphi) \xi\left(k-h_{1}\right),
\end{aligned}
$$




$$
\begin{aligned}
- & \sum_{i=-h_{2}+1}^{0} \eta^{T}(k-1+i) P_{11}(\varphi) \eta(k-1+i) \\
& \leq h_{2} \xi^{T}(k) M_{2}(\varphi) \xi(k)+2 \xi^{T}(k) N_{2}(\varphi) \sum_{i=-h_{2}+1}^{0} \eta(k-1+i) \\
& =h_{2} \xi^{T}(k) M_{2}(\varphi) \xi(k)+2 \xi^{T}(k) N_{2}(\varphi) \xi(k)-2 \xi^{T}(k) N_{2}(\varphi) \xi\left(k-h_{2}\right), \\
- & \sum_{i=-r h_{1}+1}^{0} \eta^{T}(k-1+i) P_{12}(\varphi) \eta(k-1+i) \\
& \leq r h_{1} \xi^{T}(k) M_{3}(\varphi) \xi(k)+2 \xi^{T}(k) N_{3}(\varphi) \sum_{i=-r h_{1}+1}^{0} \eta(k-1+i) \\
= & r h_{1} \xi^{T}(k) M_{3}(\varphi) \xi(k)+2 \xi^{T}(k) N_{3}(\varphi) \xi(k) \\
& -2 \xi^{T}(k) N_{3}(\varphi) \xi\left(k-r h_{1}\right), \\
- & \sum_{i=-r h_{2}+1}^{0} \eta^{T}(k-1+i) P_{13}(\varphi) \eta(k-1+i) \\
& \leq r h_{2} \xi^{T}(k) M_{4}(\varphi) \xi(k)+2 \xi^{T}(k) N_{4}(\varphi) \sum_{i=-r h_{2}+1}^{0} \eta(k-1+i) \\
= & r h_{2} \xi^{T}(k) M_{4}(\varphi) \xi(k)+2 \xi^{T}(k) N_{4}(\varphi) \xi(k) \\
& -2 \xi^{T}(k) N_{4}(\varphi) \xi\left(k-r h_{2}\right) .
\end{aligned}
$$

Therefore, we conclude that

$$
\begin{aligned}
\Delta V_{4} & (k, \xi(k)) \\
\leq & h_{1} \eta^{T}(k) P_{10}(\varphi) \eta(k)+h_{1} \xi^{T}(k) M_{1}(\varphi) \xi(k)+2 \xi^{T}(k) N_{1}(\varphi) \xi(k) \\
& -2 \xi^{T}(k) N_{1}(\varphi) \xi\left(k-h_{1}\right)+h_{2} \eta^{T}(k) P_{11}(\varphi) \eta(k) \\
& +h_{2} \xi^{T}(k) M_{2}(\varphi) \xi(k)+2 \xi^{T}(k) N_{2}(\varphi) \xi(k) \\
& -2 \xi^{T}(k) N_{2}(\varphi) \xi\left(k-h_{2}\right)+r h_{1} \eta^{T}(k) P_{12}(\varphi) \eta(k) \\
& +r h_{1} \xi^{T}(k) M_{3}(\varphi) \xi(k)+2 \xi^{T}(k) N_{3}(\varphi) \xi(k) \\
& -2 \xi^{T}(k) N_{3}(\varphi) \xi\left(k-r h_{1}\right)+r h_{2} \eta^{T}(k) P_{13}(\varphi) \eta(k) \\
& +r h_{2} \xi^{T}(k) M_{4}(\varphi) \xi(k)+2 \xi^{T}(k) N_{4}(\varphi) \xi(k) \\
& -2 \xi^{T}(k) N_{4}(\varphi) \xi\left(k-r h_{2}\right) .
\end{aligned}
$$

By Lemma 2.2, the increments of $V_{5}(k, \xi(k))$ and $V_{6}(k, \xi(k))$ can be expressed mathematically as

$$
\begin{aligned}
& \Delta V_{5}(k, \xi(k)) \\
& \quad=h_{1}^{2} \eta^{T}(k) P_{14}(\varphi) \eta(k)-h_{1} \sum_{i=k-h_{1}}^{k-1} \eta^{T}(i) P_{14}(\varphi) \eta(i)
\end{aligned}
$$




$$
\begin{aligned}
& +h_{2}^{2} \eta^{T}(k) P_{15}(\varphi) \eta(k)-h_{2} \sum_{i=k-h_{2}}^{k-1} \eta^{T}(i) P_{15}(\varphi) \eta(i) \\
& +\rho^{2} \eta^{T}(k) P_{16}(\varphi) \eta(k)-\rho \sum_{i=k-h_{2}}^{k-h_{1}-1} \eta^{T}(i) P_{16}(\varphi) \eta(i) \\
\leq & h_{1}^{2} \eta^{T}(k) P_{14}(\varphi) \eta(k)-\left(\sum_{i=k-h_{1}}^{k-1} \eta(i)\right)^{T} P_{14}(\varphi)\left(\sum_{i=k-h_{1}}^{k-1} \eta(i)\right) \\
& +h_{2}^{2} \eta^{T}(k) P_{15}(\varphi) \eta(k)-\left(\sum_{i=k-h_{2}}^{k-1} \eta(i)\right)^{T} P_{15}(\varphi)\left(\sum_{i=k-h_{2}}^{k-1} \eta(i)\right) \\
& +\rho^{2} \eta^{T}(k) P_{16}(\varphi) \eta(k)-\left(\sum_{i=k-h_{2}}^{k-h_{1}-1} \eta(i)\right)^{T} P_{16}(\varphi)\left(\sum_{i=k-h_{2}}^{k-h_{1}-1} \eta(i)\right) \\
\Delta V_{6}(k, \xi(k)) & h_{2}^{2} \eta^{T}(k) Z_{1}(\varphi) \eta(k)-h_{2} \sum_{i=k-h_{2}}^{k-1} \eta^{T}(i) Z_{1}(\varphi) \eta(i) \\
& +\left(r h_{2}\right)^{2} \eta^{T}(k) Z_{2}(\varphi) \eta(k)-r h_{2} \sum_{i=k-r h_{2}}^{k-1} \eta^{T}(i) Z_{2}(\varphi) \eta(i) \\
& +\left(\sum_{i=k-r h(k)}^{k-1} \eta(i)\right) . \\
\leq & h_{2}^{2} \eta^{T}(k) Z_{1}(\varphi) \eta(k)-\left(\sum_{i=k-h(k)}^{k-1} \eta(i)\right) Z_{1}(\varphi)\left(\sum_{i=k-h(k)}^{k-1} \eta(i)\right)
\end{aligned}
$$

The forward difference of $V_{7}(k, \xi(k))$ can be obtained as

$$
\begin{aligned}
\Delta V_{7}(k, \xi(k))= & \eta^{T}(k)\left[\frac{h_{1}^{4}}{4} P_{17}(\varphi)+\frac{\left(h_{2}-h_{1}\right)^{2}}{4} P_{18}(\varphi)\right] \eta(k) \\
& -\frac{h_{1}^{2}}{2} \sum_{i=-h_{1}}^{-1} \sum_{j=k+i}^{k-1} \eta^{T}(j) P_{17}(\varphi) \eta(j) \\
& -\frac{1}{2} \sum_{i=k-h_{2}}^{k-h_{1}-1} \sum_{j=i}^{k-h_{1}-1} \eta^{T}(j) P_{18}(\varphi) \eta(j) .
\end{aligned}
$$

By Lemma 2.3, we obtain

$$
-\frac{h_{1}^{2}}{2} \sum_{i=-h_{1}}^{-1} \sum_{j=k+i}^{k-1} \eta^{T}(j) P_{17}(\varphi) \eta(j)
$$




$$
\leq-\left[h_{1} \xi(k)-\sum_{i=k-h_{1}}^{k-1} \xi(i)\right]^{T} P_{17}(\varphi)\left[h_{1} \xi(k)-\sum_{i=k-h_{1}}^{k-1} \xi(i)\right],
$$

$-\frac{1}{2} \sum_{i=k-h_{2}}^{k-h_{1}-1} \sum_{j=i}^{k-h_{1}-1} \eta^{T}(j) P_{18}(\varphi) \eta(j)$

$$
\begin{aligned}
= & -\frac{1}{2} \sum_{i=k-h(k)}^{k-h_{1}-1} \sum_{j=i}^{k-h_{1}-1} \eta^{T}(j) P_{18}(\varphi) \eta(j)-\frac{1}{2} \sum_{i=k-h_{2}}^{k-h(k)-1} \sum_{j=i}^{k-h(k)-1} \eta^{T}(j) P_{18}(\varphi) \eta(j) \\
& -\frac{1}{2} \sum_{i=k-h_{2}}^{k-h(k)-1} \sum_{j=k-h(k)}^{k-h_{1}-1} \eta^{T}(j) P_{18}(\varphi) \eta(j) \\
\leq & -\left[\sum_{i=k-h(k)}^{k-h_{1}-1} \sum_{j=i}^{k-h_{1}-1} \eta\right]^{T} \frac{1}{\varphi^{2}(k)} P_{18}(\varphi)\left[\sum_{i=k-h(k)}^{k-h_{1}-1} \sum_{j=i}^{k-h_{1}-1} \eta(j)\right] \\
& -\left[\sum_{i=k-h_{2}}^{k-h(k)-1} \sum_{j=i}^{k-h(k)-1} \eta(j)\right]^{T} \frac{1}{\beta^{2}(k)} P_{18}(\varphi)\left[\sum_{i=k-h_{2}}^{k-h(k)-1} \sum_{j=i}^{k-h(k)-1} \eta(j)\right] \\
= & -\left[\xi\left(k-h_{1}\right)-\psi(k)\right]^{T} P_{18}(\varphi)\left[\xi\left(k-h_{1}\right)-\psi(k)\right] \\
& -[\xi(k-h(k))-\phi(k)]^{T} P_{18}(\varphi)[\xi(k-h(k))-\phi(k)] .
\end{aligned}
$$

Substituting (40) and (41) into (39), we obtain

$$
\begin{aligned}
\Delta V_{7}(k, \xi(k)) \leq & \eta^{T}(k)\left[\frac{h_{1}^{4}}{4} P_{17}(\varphi)+\frac{\left(h_{2}-h_{1}\right)^{2}}{4} P_{18}(\varphi)\right] \eta(k) \\
& -\left[h_{1} \xi(k)-\sum_{i=k-h_{1}}^{k-1} \xi(i)\right]^{T} P_{17}(\varphi)\left[h_{1} \xi(k)-\sum_{i=k-h_{1}}^{k-1} \xi(i)\right] \\
& -\left[\xi\left(k-h_{1}\right)-\psi(k)\right]^{T} P_{18}(\varphi)\left[\xi\left(k-h_{1}\right)-\psi(k)\right] \\
& -[\xi(k-h(k))-\phi(k)]^{T} P_{18}(\varphi)[\xi(k-h(k))-\phi(k)] .
\end{aligned}
$$

Taking the forward difference of $V_{8}(k, \xi(k))$, we obtain

$$
\begin{aligned}
\Delta V_{8}(k, \xi(k))= & w \xi^{T}(k) P_{19}(\varphi) \xi(k) \\
& -\frac{1}{w}\left[\sum_{i=1}^{\infty} \delta(i) \xi(k-i)\right]^{T} P_{19}(\varphi)\left[\sum_{i=1}^{\infty} \delta(i) \xi(k-i)\right] .
\end{aligned}
$$

From (3) and (4), for any scalars $\epsilon_{1}, \epsilon_{2}>0$, we obtain

$$
\begin{aligned}
& \epsilon_{1}\left(\varphi^{2} \xi^{T}(k) \xi(k)-f_{1}^{T}(k, \xi(k)) f_{1}(k, \xi(k))\right) \geq 0, \\
& \epsilon_{2}\left(\beta^{2} \xi^{T}(k-h(k)) \xi(k-h(k))-f_{2}^{T}(k, \xi(k-h(k))) f_{2}(k, \xi(k-h(k)))\right) \geq 0 .
\end{aligned}
$$

It follows from (22)-(45) that

$$
\Delta V(k, \xi(k)) \leq \zeta^{T}(k) \sum_{i=1}^{N} \sum_{j=1}^{N} \varphi_{i} \varphi_{j} \prod_{i, j} \zeta(k)
$$


where $\zeta^{T}(k)=\left[\xi^{T}(k) \eta^{T}(k) \xi^{T}\left(k-h_{1}\right) \xi^{T}\left(k-h_{2}\right) \xi^{T}(k-h(k)) \xi^{T}\left(k-r h_{1}\right) \times\right.$ $\xi^{T}\left(k-r h_{2}\right) \xi^{T}(k-r h(k)) \sum_{i=k-h_{1}}^{k-1} \xi^{T}(i) \sum_{i=k-r h_{1}}^{k-1} \xi^{T}(i) \sum_{i=k-h_{2}}^{k-1} \xi^{T}(i) \sum_{i=k-r h_{2}}^{k-1} \xi^{T}(i) \times$ $\sum_{i=k-h_{2}}^{k-h_{1}-1} \xi^{T}(i) \sum_{i=k-r h_{2}}^{k-r h_{1}-1} \xi^{T}(i) \sum_{i=k-h_{1}}^{k-1} \eta^{T}(i) \sum_{i=k-r h_{1}}^{k-1} \eta^{T}(i) \sum_{i=k-h_{2}}^{k-1} \eta^{T}(i) \sum_{i=k-r h_{2}}^{k-1} \eta^{T}(i) \quad \times$ $\sum_{i=k-h_{2}}^{k-h_{1}-1} \eta^{T}(i) \sum_{i=k-r h_{2}}^{k-r h_{1}-1} \eta^{T}(i) \sum_{i=k-h(k)}^{k-1} \eta^{T}(i) \sum_{i=k-r h(k)}^{k-1} \eta^{T}(i) \psi(k) \phi(k) f_{1}(k, \xi(k)) f_{2}(k, \xi(k \quad-$ $\left.h(k))) \sum_{i=1}^{\infty} \delta(i) \xi(k-i)\right]$ and $\prod_{i, j}$ is defined as in (6). The fact that $\sum_{i=1}^{N} \varphi_{i}=1$ leads to the following identities:

$$
\begin{aligned}
& \sum_{i=1}^{N} \sum_{j=1}^{N} \varphi_{i} \varphi_{j} \prod_{i, j}=\sum_{i=1}^{N} \varphi_{i}^{2} \prod_{i, i}+\sum_{i=1}^{N-1} \sum_{j=i+1}^{N} \varphi_{i} \varphi_{j}\left[\prod_{i, j}+\prod_{j, i}\right], \\
& (N-1) \sum_{i=1}^{N} \varphi_{i}^{2}-2 \sum_{i=1}^{N-1} \sum_{j=i+1}^{N} \varphi_{i} \varphi_{j}=\sum_{i=1}^{N-1} \sum_{j=i+1}^{N}\left[\varphi_{i}-\varphi_{j}\right]^{2} \geq 0 .
\end{aligned}
$$

By (46)-(48), if (7)-(10) are feasible, then

$$
\Delta V(k, \xi(k))<-\omega\|\xi\|^{2}
$$

where $\omega>0$. Therefore, system (1)-(2) is robustly stable. This completes the proof.

Remark 1 Theorem 3.1 reduces to LMIs stability condition for discrete-time system with time-varying delays and nonlinear uncertainties [21] if $A(\varphi)=A, B(\varphi)=B, C(\varphi)=0$ where $A, B \in R^{n \times n}$. We use the mixed model transformation in this paper, and utilize the various inequalities to reduce the possible conservatism of the obtained condition.

Remark 2 If $A(\varphi)=A, B(\varphi)=B, C(\varphi)=0$ and $f_{1}(k, \xi(k))=f_{2}(k, \xi(k-h(k)))=0$ where $A, B \in$ $R^{n \times n}$, model (1) is simplified to the discrete-time system with time-varying delays studied in $[2,4,6,7,16,18]$. So the investigation in this paper expands and improves the studies in the literature.

\section{Numerical examples}

Example 4.1 Consider the system

$$
\xi(k+1)=A \xi(k)+B \xi(k-h(k))+f_{1}(k, \xi(k))+f_{2}(k, \xi(k-h(k)))
$$

with the parameters

$$
A=\left[\begin{array}{cc}
0.80 & 0 \\
0.05 & 0.90
\end{array}\right], \quad B=\left[\begin{array}{cc}
-0.10 & 0 \\
-0.20 & -0.10
\end{array}\right], \quad \gamma \geq 0, \beta \geq 0,
$$

which were considered in [21].

Decompose the matrices $A$ and $B$ as $A=A_{1}+A_{2}$ and $B=B_{1}+B_{2}$, where

$$
\begin{array}{rlr}
A_{1} & =\left[\begin{array}{cc}
-0.5 & -0.1 \\
-0.02 & -0.3
\end{array}\right], & A_{2}=\left[\begin{array}{cc}
1.3 & 0.1 \\
0.07 & 1.2
\end{array}\right], \\
B_{1}=\left[\begin{array}{ll}
-0.5 & -0.1 \\
-0.3 & -0.4
\end{array}\right], & B_{2}=\left[\begin{array}{ll}
0.4 & 0.1 \\
0.1 & 0.3
\end{array}\right] .
\end{array}
$$


Table 1 The maximum upper bounds of $h_{2}$ for Example 4.1

\begin{tabular}{lccccc}
\hline Method & $h_{1}$ & 2 & 6 & 10 & 14 \\
\hline$\gamma=0, \beta=0$ & & & & & \\
Ramakrishnan and Ray (2013) [21] & $h_{2}$ & 17 & 18 & 20 & 22 \\
Ours & $h_{2}$ & 57 & 57 & 57 & 57 \\
$\gamma=0, \beta=0.1$ & & & & & \\
Ramakrishnan and Ray (2013) [21] & $h_{2}$ & 11 & 13 & 15 & 17 \\
Ours & $h_{2}$ & 57 & 57 & 57 & 57 \\
$\gamma=0.1, \beta=0.1$ & & & & & 11 \\
Ramakrishnan and Ray (2013) [21] & $h_{2}$ & 10 & 11 & 57 & 57 \\
Ours & $h_{2}$ & 57 & 57 & 57 & \\
\hline
\end{tabular}

Table 2 The maximum upper bounds of $h_{2}$ for Example 4.2

\begin{tabular}{lllll}
\hline$h_{1}$ & 4 & 10 & 20 & 30 \\
\hline Peng (2012) [18] & 18 & 20 & 26 & 35 \\
Feng et al. (2015) [2] & 21 & 22 & 27 & 35 \\
Kwon et al. (2013) [7] & 22 & 23 & 27 & 36 \\
Kim (2015) [6] & 22 & 23 & 28 & 36 \\
Nam et al. (2015) [16] & 22 & 23 & 29 & 36 \\
Hien and Trinh (2016) [4] & 27 & 28 & 35 & 39 \\
Ours & 56 & 56 & 56 & 56 \\
\hline
\end{tabular}

Applying Theorem 3.1 to system (50)-(51), the numerical results of our criterion are dramatically less conservative than those of [21], as shown in Table 1.

Example 4.2 Consider the system

$$
\xi(k+1)=A \xi(k)+B \xi(k-h(k))
$$

with the parameters

$$
A=\left[\begin{array}{cc}
0.80 & 0 \\
0.05 & 0.90
\end{array}\right], \quad B=\left[\begin{array}{cc}
-0.10 & 0 \\
-0.20 & -0.10
\end{array}\right] \text {, }
$$

which were considered in $[2,4,6,7,16,18]$.

Decompose the matrices $A$ and $B$ as $A=A_{1}+A_{2}$ and $B=B_{1}+B_{2}$, where

$$
\begin{array}{ll}
A_{1}=\left[\begin{array}{cc}
-0.4 & -0.1 \\
-0.02 & -0.3
\end{array}\right], & A_{2}=\left[\begin{array}{cc}
1.2 & 0.1 \\
0.07 & 1.2
\end{array}\right], \\
B_{1}=\left[\begin{array}{ll}
-0.5 & -0.1 \\
-0.3 & -0.4
\end{array}\right], & B_{2}=\left[\begin{array}{ll}
0.4 & 0.1 \\
0.1 & 0.3
\end{array}\right] .
\end{array}
$$

Given various values of $h_{1}$, the obtained upper bounds for $h_{2}$ are shown in Table 2. The numerical results show the asymptotic stability of system (52) with (53). Moreover, by applying Theorem 3.1, less conservative results are achieved.

\section{Conclusions}

The problem of delay-interval-dependent robust stability for LPD discrete-time system with mixed time-varying delays and nonlinear uncertainties was studied. By utilizing var- 
ious inequalities, a mixed model transformation, zero equations, and a new parameterdependent Lyapunov-Krasovskii functional, the new delay-interval-dependent stability criterion is derived and formulated in terms of LMIs for the system. Some numerical examples are also illustrated to exhibit the effectiveness with less conservatism of the proposed stability criterion.

\section{Acknowledgements}

The author would like to thank the editors and reviewers for careful reading of the original manuscript and for their valuable suggestions.

\section{Funding}

This work was supported by the Faculty of Science at Sriracha, Kasetsart University Sriracha Campus, Thailand (Grant number P003/2018); the Thailand Research Fund (TRF); the Office of the Higher Education Commission (OHEC), Khon Kaen University (Grant number MRG6080042); Research and Academic Affairs Promotion Fund, Faculty of Science, Khon Kaen University, Fiscal Year 2019; and National Research Council of Thailand and Khon Kaen University, Thailand (Grant number 6200069).

\section{Availability of data and materials}

Not applicable.

\section{Competing interests}

The authors declare that they have no competing interests.

Authors' contributions

All authors read and approved the final manuscript

\section{Author details}

'Department of Mathematics, Faculty of Science, Khon Kaen University, Khon Kaen, Thailand. ${ }^{2}$ Faculty of Science at Sriracha, Kasetsart University Sriracha Campus, Chon Buri, Thailand.

\section{Publisher's Note}

Springer Nature remains neutral with regard to jurisdictional claims in published maps and institutional affiliations.

Received: 30 January 2019 Accepted: 4 June 2019 Published online: 21 June 2019

\section{References}

1. Botmart, T., Niamsup, P.: Robust exponential stability and stabilizability of linear parameter dependent systems with delays. Appl. Math. Comput. 217(6), 2551-2566 (2010)

2. Feng, Z., Lam, J., Yang, G.H.: Optimal partitioning method for stability analysis of continuous/discrete delay systems. Int. J. Robust Nonlinear Control 25(4), 559-574 (2015)

3. Gao, H., Chen, T.W.: New results on stability of discrete-time systems with time-varying state delay. IEEE Trans. Autom. Control 52(2), 328-334 (2007)

4. Hien, L.V., Trinh, H.: New finite-sum inequalities with applications to stability of discrete time-delay systems. Automatica 71, 197-201 (2016)

5. Jiang, X., Han, Q., Yu, X.: Stability criteria for linear discrete-time systems with interval-like time-varying delay. In: Proceedings of the American Mathematical Society, pp. 2817-2822 (2005)

6. Kim, S.H.: Further results on stability analysis of discrete-time systems with time-varying delays via the use of novel convex combination. Appl. Math. Comput. 261, 104-113 (2015)

7. Kwon, O.M., Park, M.J., Park, J.H., Lee, S.M., Cha, E.J.: Stability and stabilization for discrete-time systems with time-varying delays via augmented Lyapunov-Krasovskii functional. J. Franklin Inst. 350(3), 521-540 (2013)

8. la Sen, M.D.: Robust stabilization of a class of polytopic linear time-varying continuous systems under point delays and saturating controls. Appl. Math. Comput. 181(1), 73-83 (2006)

9. Li, Y.: Impulsive synchronization of stochastic neural networks via controlling partial states. Neural Process. Lett. 46(1), 56-69 (2017)

10. Li, Y., Li, B., Liu, Y., Lu, J., Wang, Z., Alsaadi, F.E.: Set stability and stabilization of switched Boolean networks with state-based switching. IEEE Access $635624-35630$ (2018)

11. Li, Y., Lou, J., Wang, Z., Alsaadi, F.E.: Synchronization of dynamical networks with nonlinearly coupling function under hybrid pinning impulsive controllers. J. Franklin Inst. 355 6520-6530 (2018)

12. Maharajan, C., Raja, R., Cao, J., Rajchakit, G., Alsaedi, A.: Novel results on passivity and exponential passivity for multiple discrete delayed neutral-type neural networks with leakage and distributed time-delays. Chaos Solitons Fractals 115 , 268-282 (2018)

13. Mukdasai, K.: Robust exponential stability for Ipd discrete-time system with interval time-varying delay. J. Appl. Math. 2012, Article ID 237430 (2012)

14. Mukdasai, K., Niamsup, P.: Robust stability of discrete-time linear parameter dependent system with delay. Thai J. Math. 8, 11-20 (2010). Special Issue (Annual Meeting in Mathematics)

15. Nam, P.T., Hien, H.M., Phat, V.N.: Asymptotic stability of linear state-delayed neutral systems with polytope type uncertainties. Dyn. Syst. Appl. 19, 63-72 (2010) 
16. Nam, P.T., Pubudu, P.N., Trinh, H.: Discrete Wirtinger-based inequality and its application. J. Franklin Inst. 352 1893-1905 (2015)

17. Niamsup, P., Rajchakit, G.: New results on robust stability and stabilization of linear discrete-time stochastic systems with convex polytopic uncertainties. J. Appl. Math. 2013, Article ID 368259 (2013)

18. Peng, C.: Improved delay-dependent stabilisation criteria for discrete systems with a new finite sum inequality. IET Control Theory Appl. 6(3), 448-453 (2012)

19. Phat, V.N., Nam, P.T.: Exponential stability and stabilization of uncertain linear time-varying systems using parameter dependent Lyapunov function. Int. J. Control 80(8), 1333-1341 (2007)

20. Raja, R., Raja, U.K., Samidurai, R., Leelamani, A.: Dissipativity of discrete-time BAM stochastic neural networks with Markovian switching and impulses. J. Franklin Inst. 350(10), 3217-3247 (2013)

21. Ramakrishnan, K., Ray, G.: Robust stability criteria for a class of uncertain discrete-time systems with time-varying delay. Appl. Math. Model. 37(3), 1468-1479 (2013)

22. Sowmiya, C., Raja, R., Cao, J., Li, X., Rajchakit, G.: Discrete-time stochastic impulsive BAM neural networks with leakage and mixed time delays: an exponential stability problem. J. Franklin Inst. 355(10), 4404-4435 (2018)

23. Sowmiya, C., Raja, R., Cao, J., Rajchakit, G., Alsaedi, A.: Enhanced robust finite-time passivity for Markovian jumping discrete-time BAM neural networks with leakage delay. Adv. Differ. Equ. 2017, 318 (2017)

24. Sowmiya, C., Raja, R., Zhu, Q., Rajchakit, G.: Further mean-square asymptotic stability of impulsive discrete-time stochastic BAM neural networks with Markovian jumping and time-varying delays. J. Franklin Inst. 356(1), 561-591 (2019)

25. Udpin, S., Niamsup, P.: Robust stability of discrete-time LPD neural networks with time-varying delay. Commun. Nonlinear Sci. Numer. Simul. 14, 3914-3924 (2009)

26. Wang, T., Xue, M.X., Fei, S.M., Li, T.: Triple Lyapunov functional technique on delay-dependent stability for discrete-time dynamical networks. Neurocomputing 122, 221-228 (2013)

27. Wang, T., Xue, M.X., Zhang, C., Fei, S.M.: Improved stability criteria on discrete-time systems with time-varying and distributed delays. Int. J. Autom. Comput. 10(3), 260-266 (2013)

28. Xia, Y., Jia, Y.: Robust stability functionals of state delayed systems with polytopic type uncertain via parameter-dependent Lyapunov functions. Int. J. Control 75, 1427-1434 (2002)

29. Zhang, W., Su, H.S., Han, Z.Z.: Robust stability for nonlinear discrete-time systems with interval time-varying delay. In: 2011 Third International Conference on Measuring Technology and Mechatronics Automation, pp. 191-194 (2011)

30. Zhang, X., Han, Q.L.: A new finite sum inequality approach to delay-dependent $h_{\infty}$ control of discrete-time systems with time-varying delay. Int. J. Robust Nonlinear Control 18(6), 630-647 (2008)

\section{Submit your manuscript to a SpringerOpen ${ }^{\circ}$ journal and benefit from:}

- Convenient online submission

- Rigorous peer review

- Open access: articles freely available online

- High visibility within the field

- Retaining the copyright to your article

Submit your next manuscript at $\boldsymbol{\triangleright}$ springeropen.com 